\title{
PERFORMANCE OF A RADIO-FREQUENCY-BASED STREAK CAMERA
}

\author{
A.V. Aleksandrov ${ }^{1)}$, N.S. Dikansky ${ }^{1)}$, V. Guidi' ${ }^{2)}$, G.V. Lamanna ${ }^{3)}$, P.V. Logatchov ${ }^{1)}$, S.V. \\ Shiyankov ${ }^{1)}$, L. Tecchio ${ }^{4)}$ \\ ${ }^{1)}$ Institute of Nuclear Physics, 630090 Novosibirsk, Russia. \\ ${ }^{2)}$ Dipartimento di Fisica dell'Università and INFN, I-44100 Ferrara, Italy. \\ ${ }^{3)}$ Dipartimento di Fisica dell'Università and INFN, I-70125 Bari, Italy \\ ${ }^{4)}$ Laboratori Nazionali di Legnaro INFN, I-35020 Legnaro, Italy.
}

\section{Abstract}

We have experimented with a streak camera based on radio-frequency deflection of the electron beam. The time resolution was measured to lie in the sub-ps domain. The method also allowed considerable simplification of triggering, weaker dependence on space-charge within the electron beam, high modularity, and ease of implementation. We discuss experimental results and future prospects.

\section{INTRODUCTION}

This paper is devoted to illustrate the setting up of a RFbased streak Camera (RFC). Beam deflection was accomplished by replacing the standard deflecting plates of a conventional streak camera (SC) by a RF cavity located just after the accelerating optics.

The magnetic field in the cavity imparts a transverse force to incoming electrons, whose direction depends on the time at which each individual electron enters the cavity, i.e. on the RF-phase experienced by an incoming electron (circular scanning) [1]. The high sweep speed, which is possible by the method, envisaged sub-ps resolution [2]. The other components of a streak camera keep unchanged.

The circular deflection of an electron beam can be performed by transverse magnetic field of $T M_{110}$ mode with circular polarization in a cylindrical cavity. Circular polarization is provided by exciting two orthogonal modes shifted by $\pi / 2$ rad in phase.

A complete treatment on theoretical basis for the RFC can be found in Ref. [3].

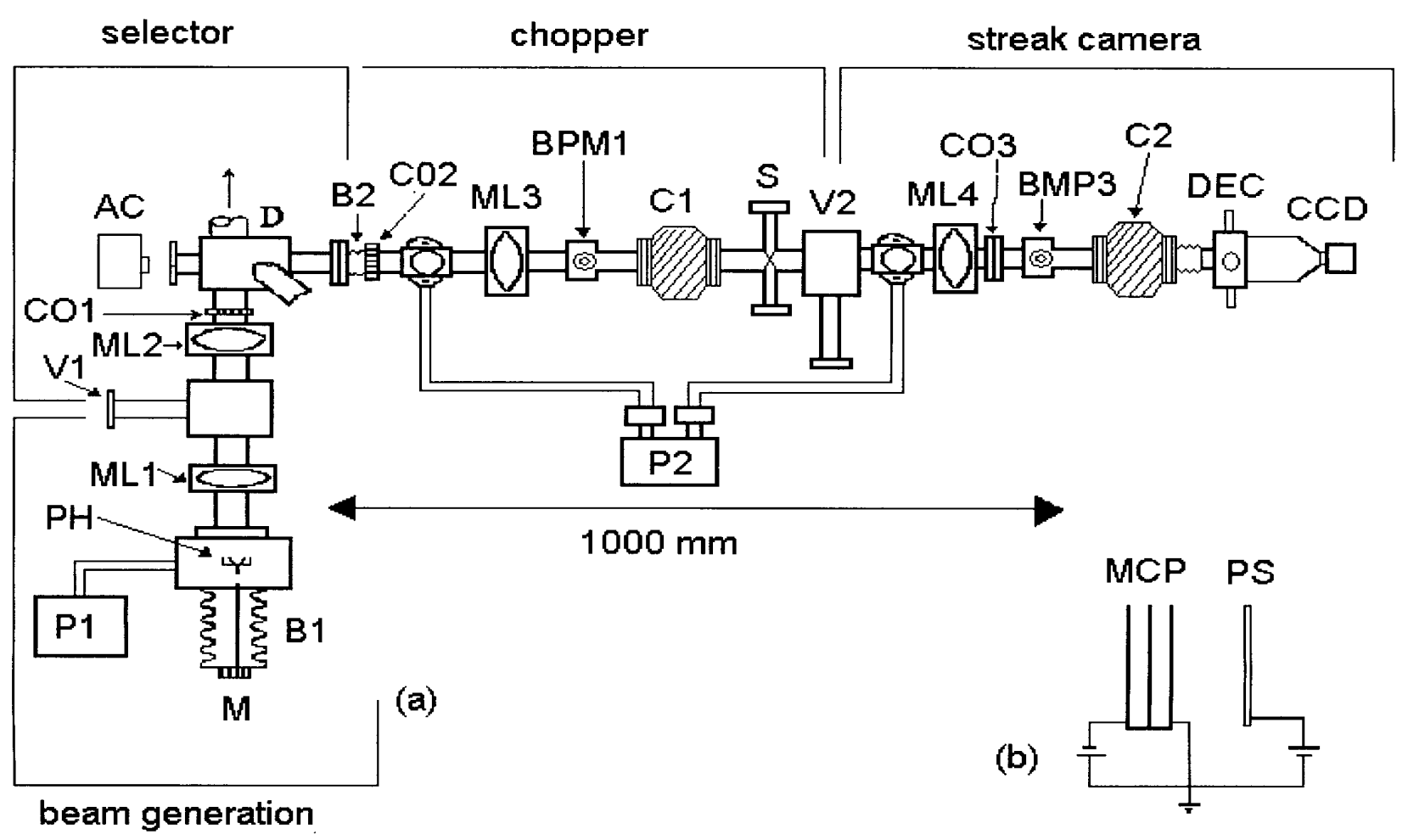

Figure 1 Schematic drawing of the experimental apparatus 


\section{EXPERIMENTAL}

The setup of the experiment is sketched in Fig. 1. It basically consisted of three parts: electron gun, choppersystem, and RFC.

In order to test the time resolution of the RFC, one had to provide a sub-picosecond electron bunch. Since the existing GaAs photo-gun operated at $40 \mathrm{kV}$ [4] was unable to deliver electron bunches shorter than $60 \mathrm{ps}$, a system to form a sub-ps electron bunch was implemented. This was a chopper consisting of deflecting cavity $C l$, drift tube, and slit $S$ (see Fig.1(a)). An iris with $400 \mu \mathrm{m}$ aperture was installed to reduce transverse beam size at the entrance. The slit width of the chopper could be varied through micrometric control.

The deflecting cavity was axially symmetric and made of OFHC copper. The electromagnetic field of the $T M_{110}$ mode inside the cavity was excited through magnetic loop. The bunch outcoming the cavity was swept on the horizontal plane and hit onto a vertical slit. In our case a minimum beam size of about $200 \mu \mathrm{m}$ provided a minimum bunch length of $0.7 \mathrm{ps}$ at the exit of the chopper system. This way the electron bunch passing through the chopper cavity was sufficiently short to calibrate the RFC.

The measuring part of the experimental apparatusstrictly speaking - the RFC consisted of deflecting cavity $C 2$, drift tube, detector $D E C$ and magnetic lens $M L 4$. Cavity $\mathrm{C} 2$ had the same design as the chopper cavity $(\mathrm{Cl})$ but was fed by two orthogonal $T M_{110}$ modes, resulting in a rotating magnetic field orthogonal to the cavity axis. Each mode was excited by separate magnetic loop. The two loops were geometrically at right angle and fed by two RF amplifiers, reciprocally shifted by $\pi / 2 \mathrm{rad}$ in phase. The resonant frequency of each mode could be separately adjusted by means of two piston tuners.

Deflected electrons passed through the drift tube and were collected by position sensitive detector. This was a two-stage MCP coupled to a circular phosphor screen 28 $\mathrm{mm}$ in diameter (see Fig.1(b)). High voltage of $4 \mathrm{kV}$ was applied between the backside of the MCP and the screen. The image on the screen was read out by a charge coupled device (CCD) camera and acquired by computer. The overall resolution of the detector was about $60 \mu \mathrm{m}$ (rms) mainly limited by the MCP.

A pulsed RF-power amplifier with three separate channels was used to feed both the chopper and the measuring cavities. Output power up to $800 \mathrm{~W}$ could be independently controlled on each channel. Phase difference between the two channels could be varied by coaxial phaseshifter. The signal of the mode-locking RF generator of the laser was multiplied by 64 in frequency and used as an input for the amplifier. This scheme provided synchronization between electron bunch and chopper phase.

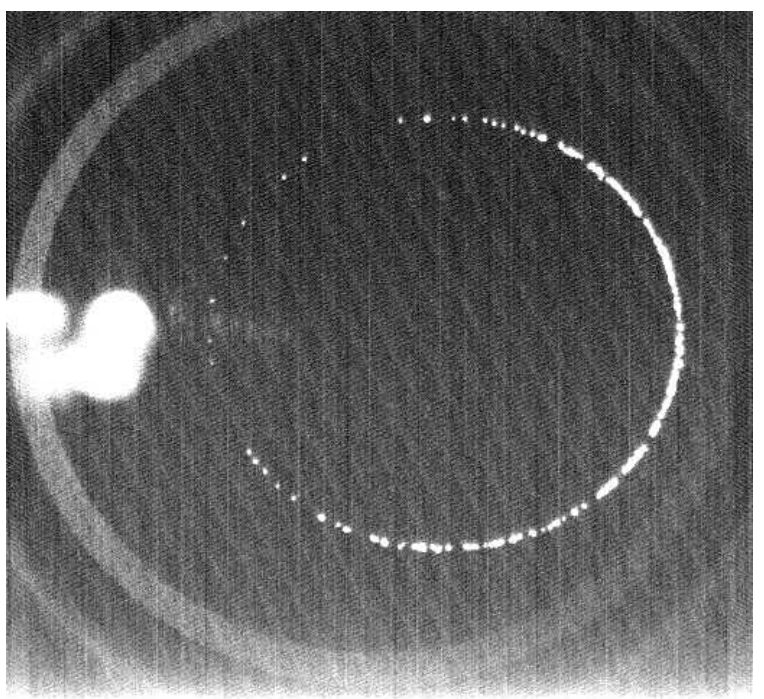

Figure 2. Image of a circularly scanned beam on the phosphor screen. The chopper cavity is switched off.
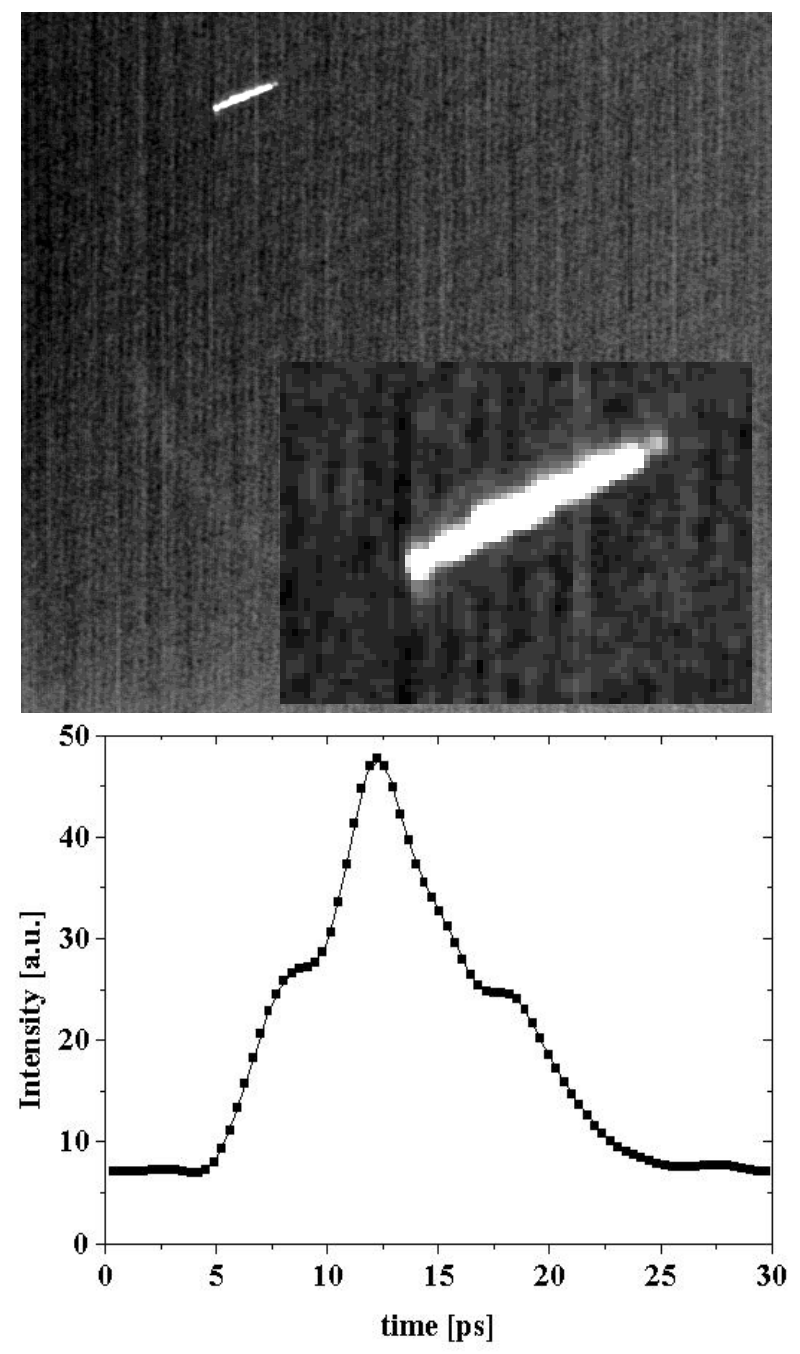

Figure 3. Image of the electron beam on the phosphor screen (magnified in the inset) and its cross-section when the chopper cavity is switched on. 


\section{RESULTS}

We firstly adjusted the optics to focalize the beam as much as possible onto the screen. It was measured that the profile of the spot exhibited an rms size of 1.7 pixels, i.e. about $100 \mu \mathrm{m}$-limited by beam emittance.

Then the RF power was fed on the cavity and phase shift between the two modes was optimized to achieve an arc of a circumference for the beam trace as shown in Fig. 2. The arc was not a complete circumference because the laser pulse was shorter than one RF period. The radius of deflection drawn by electrons on the screen is an important parameter since it is bounded up to the resolution of the instrument. Measuring the radius allowed one to calibrate the RFC: here a diameter of 314 pixels was measured, corresponding to $18.5 \mathrm{~mm}$.

The response of the RFC to a short electron bunch was tested using the chopper system and the adjustable slit. As the RF power on the chopper cavity was supplied, short electron bunches entered the RFC and were analyzed as shown in Fig. 3, where a beam-trace image and its crosssection are shown. The resolution of the instrument was sufficiently high to follow the profile of the electron bunch.

The ultimate capability of the RFC in terms of resolution can be determined by probing through even shorter electron bunches. As the RF power on the chopper cavity was raised, the outcoming electron bunch became shorter, like illustrated in Fig. 4. Error bars are rms values of the distribution obtained after a large number of measurements. The dashed line represents the resolution limit achievable by the instrument in the present configuration, i.e. $700 \mathrm{fs}$ (rms).

\section{DISCUSSION}

On the basis of the experience we gained during execution of measurements, the RFC exhibits the following advantages:

1) Microsecond accuracy for triggering is needed in contrast with normal SCs where triggering may often be a serious problem. In addition the position of the trace on the screen may provide information on the phase of the incoming bunch with respect to the RF field.

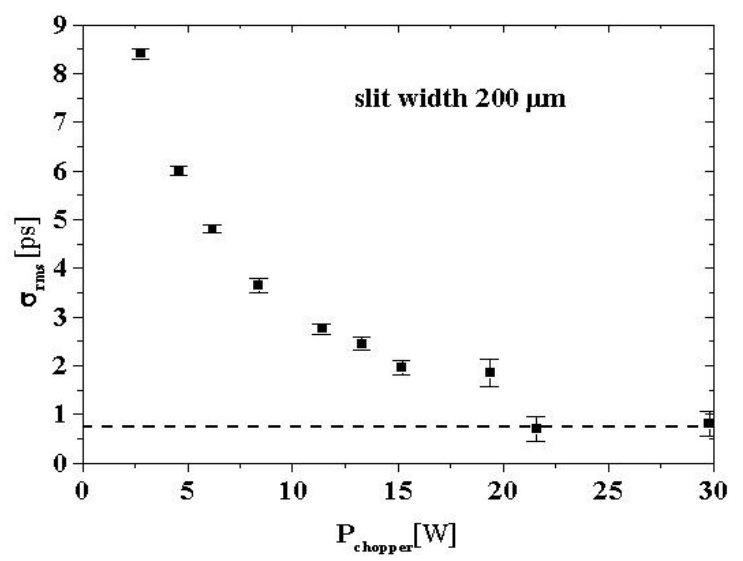

Figure 4. Dependence of bunch length on RF power

2) The accelerating voltage of conventional SC is usually no higher than $20 \mathrm{kV}$. This means that space charge may have non-negligible effect and limit the performance of a SC. On the contrary, since electron deflection is driven by magnetic field for the RFC, a bending force proportional to the bunch velocity is exerted. Operation up to $60 \mathrm{kV}$ was proven, leading to significantly weaker dependence on space charge.

3) The RFC consists of separate modules: electron source, electron gun, deflecting system and detector. Each module can be conceived to be a part of a general-purpose instrument.

4) The RFC was shown to feature a resolution no worse than $700 \mathrm{fs}$ (rms). However it is far from any physical limitation and further improvement should be obtained by increasing the angle of deflection or the screen size. Alternatively one may resort to higher frequency cavities.

A newly designed compact RFC based on the same RF cavity as we used is currently under test (Fig. 5). Expected resolution should be lower than that we measured due to better beam quality.

\section{REFERENCES}

[1] A.V. Aleksandrov et al., Rev. Sci. Instrum. 66 (1995) 3363

[2] V. Guidi et al., Meas. Sci. Tech. 6 (1995) 1555

[3] A.V. Aleksandrov et al., submitted

[4] A.V. Aleksandrov et al., Phys. Rev. E. 51 (1995) 1449.
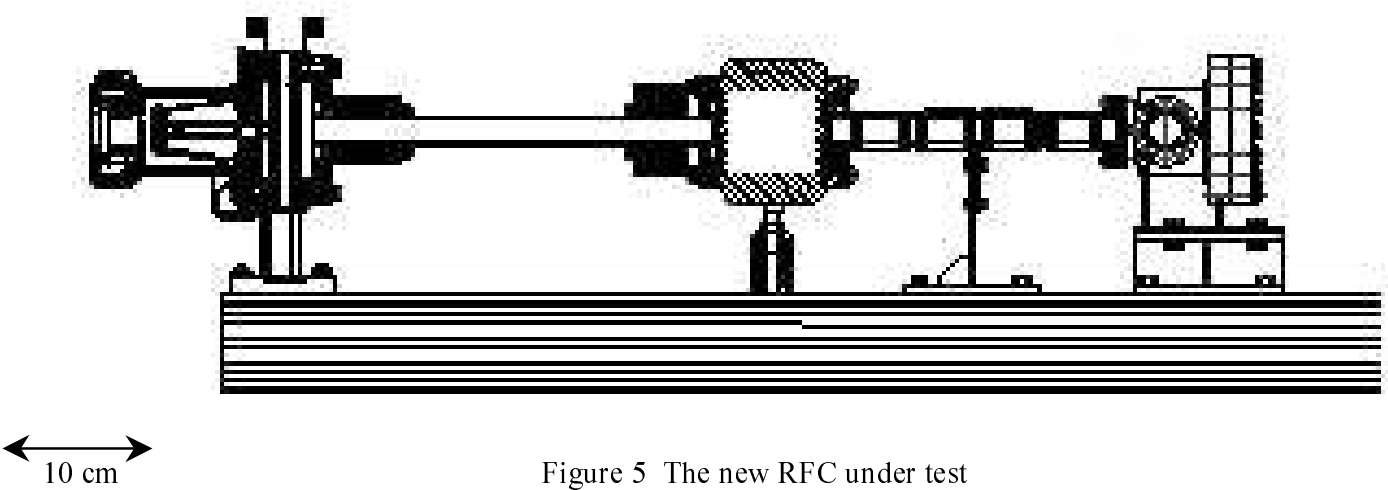

Figure 5 The new RFC under test 